\title{
The Emblems of Sleep Societies in Asia and the Republic of Korea
}

\author{
Keun Tae Kim ${ }^{1}$, Won Chul Shin ${ }^{2}$, Jee Hyun Kim ${ }^{3}$, Yong Won Cho ${ }^{1}$ \\ ${ }^{1}$ Department of Neurology, Keimyung University School of Medicine, Daegu, \\ ${ }^{2}$ Department of Neurology, Kyung Hee University School of Medicine, Seoul, \\ ${ }^{3}$ Department of Neurology, Ewha Womans University Seoul Hospital, Seoul, Korea \\ 대한민국과 아시아의 수면 관련 학회의 문장 \\ 김근태 ${ }^{1}$, 신원철 ${ }^{2}$, 김지현 ${ }^{3}$, 조용원 ${ }^{1}$ \\ ${ }^{1}$ 계명대학교 의과대학 신경과학교실, ${ }^{2}$ 경희대학교 의과대학 신경과학교실, ${ }^{3}$ 이화여자대학교 서울병원 신경과
}

Received June 19, 2021

Accepted August 2, 2021

Address for correspondence

Yong Won Cho, MD

Department of Neurology,

Keimyung University

School of Medicine,

1035 Dalgubeol-daero,

Dalseo-gu, Daegu 42601, Korea

Tel: +82-53-258-4379

Fax: +82-53-258-4380

E-mail: neurocho@gmail.com
An emblem is a symbolic representational image that stands for a certain organization, concept, team, or society. This study investigated the emblems of the sleep societies in South Korea and the international sleep societies in which they were registered as members. Three South Korean sleep societies were found by searching for the keyword 'sleep' in the Korea Citation Index. Subsequently, we identified three international societies in which the three South Korean conferences participate. The emblems can be classified according to their composition. Taegeuk patterns represent yin and yang, electroencephalography that stands for the objective indicator of sleep, and the acronym or abbreviation indicating the name of the society. All emblems in this study were combinations of pictorial images and letters. The pictorial image of the Korean Sleep Research Society is the only emblem representing an inset with Hangeul. The emblem is a medium that conveys diverse meanings beyond representation. The societies have attempted to embody the identity as well as their directions.

J Sleep Med 2021;18(2):61-65

\section{서 론}

학회(學會, society)의 사전적 의미는 "학문을 깊이 있게 연 구하고 더욱 발전하게 하기 위하여 공부하는 사람들이 만든 모임”이다. ${ }^{1}$ 세계적으로 다양한 의학회가 조직되어 활동하고 있으며, 수면의학과 관련해서는 미국을 필두로 유럽, 아시 아, 오세아니아 등의 다양한 나라에서 많은 연구자들이 활발 하게 활동하고 있다. PubMed에서 수면에 대한 연구 논문들 은 1810 년부터 검색되고 있는데, ${ }^{2}$ 관련 연구에서 가장 많이 인용된 100 개의 수면 관련 연구 중 42개가 2000년대에 집중 되어 있고 현재도 많은 수의 연구 논문이 다양한 수면의학 영역에서 생산되고 있다. ${ }^{3}$ 수면의학의 영역 확장과 수준 향상 은 세계 수면의학자들에 의한 수면학회의 구성과 활발한 교

This is an Open Access article distributed under the terms of the Creative Commons Attribution Non-Commercial License (https://creativecommons.org/licenses/by-nc/4.0) which permits unrestricted non-commercial use, distribution, and reproduction in any medium, provided the original work is properly cited.
류가 중요하게 작용하였을 것이다. 수면 관련 학회 중에서 세 계적 범위를 가진 학회라면 세계수면학회(World Sleep Society, WSS)를 들 수 있으며, 이 학회는 2016년에 세계수면연 맹(World Sleep Federation, WSF)과 세계수면협회(World Association of Sleep Medicine, WASM)가 통합되어 설립된 단체로서, ${ }^{4}$ 2020년 현재 43 개의 각국 수면학회가 회원으로 가입되어 있다. ${ }^{5}$ 이렇듯 학회들은 세계의 또는 각국의 수면 의학과 수면 연구의 발전을 위하여 조직되어 운영되고 있다.

문장(紋章, emblem)이란 국가나 단체 등을 나타내기 위하 여 사용하는 상징적인 표지로서, ${ }^{6}$ 주로 도안한 그림으로 만들 어져 있다. 우리 주변에서는 회사, 스포츠팀, 사회단체, 국가 등에서 자신들의 정체성을 나타내기 위하여 고유한 문장을 고안 또는 채택하여 사용하는 것을 쉽게 찾아볼 수 있다. 예 컨대, 우리나라 정부의 문장은 Table 1과 같다. ${ }^{7,8}$

다양한 국가, 다양한 문화 사이에서도 공통된 주제나 비슷 한 목표를 갖는 학회를 찾아볼 수 있는데, 저마다의 정체성과 
추구하는 목표와 가치를 담는 문장을 채택하여 사용하고 있 다. 본 연구에서는 우리나라의 수면 관련 학회와 이들이 회 원으로 등록된 국제 수면학회의 문장들의 의미를 알아보고, 간단한 연혁과 함께 정리해보았다.

\section{본 론}

우리나라의 공식적인 수면 관련 의학회를 찾기 위하여 한 국학술지인용색인(www.kci.go.kr)에서 제공하는 기관정보검 색의 학회명에서 '수면'을 키워드로 검색하였다. 이 결과 대한 수면학회(Korean Society of Sleep Medicine, KSSM), 대한수 면연구학회(Korean Sleep Research Society, KSRS), 대한수 면의학회(Korean Academy of Sleep Medicine, KASMED)의 3 개 학회를 확인하였다. 각 학회의 연혁과 문장에 대한 정보 를 확인하기 위하여 한국학술지인용색인에서 제공하는 기관 정보를 확인하였으며, 충분치 않은 경우에는 각 학회의 홈페 이지에서 내용을 확인하였다. 또한 각 학회의 문장과 그에 대 한 설명은 학회 홈페이지와 학회 사무국 또는 학회 임원 등의 관계자에게 메일이나 전화, 직접 만남을 통하여 확인하였다.

우리나라가 속한 국제 수면 관련 학회는 WWS, 아시아수 면학회(Asian Society of Sleep Medicine, ASSM), 아시아수 면연구학회(Asian Sleep Research Society, ASRS)를 선정하 였으며, 이들 학회의 문장은 각 학회의 홈페이지에서 수집하 였다. 이 정보가 충분치 않은 경우에는 역시 각 학회의 문장

Table 1. The Emblem of the Government Organization of the Republic of Korea ${ }^{7,8}$

Emblem
Meanings The emblem conveys a dynamic expression of
Taegeuk (the pattern forming the center of the
Korean national flag) through the unity of blue,
red, and white.
It represents the Korean government's dedication to
creating a bright future by the ideal integration of
the nation and its people of Korea and the rest of
the world, and of the past and the present together.

과 그에 대한 설명을 학회 홈페이지와 학회 사무국 또는 학회 관계자에게 메일을 통하여 확인하였다.

\section{우리나라의 수면 관련 의학회}

대한수면학회는 2006년에 창립되었으며, 여러 임상과목의 의사들로 구성된 통합학회의 성격을 띠고 있다. ${ }^{9}$ 이 학회의 영문명은 Korean Society of Sleep Medicine이며, 약자는 $\mathrm{KSSM}$ 이다. 대한수면학회의 정회원은 만 65세 이하인 수면 의학에 종사하는 의사, 전문의 수련과정을 거친 치과의사, 평 의원회에서 인정한 수면 관련 분야 박사 학위 소지자 또는 이 에 상응하는 자이다. 대한수면학회에서 현재 사용하는 문장은 2018년 10월 25일에 개정된 것이며, 큰 원 안의 대문자 S 모 양은 sleep의 첫 번째 알파벳이며, 학회명의 약자인 KSSM에 서 세 번째의 알파벳인 $\mathrm{S}$ 를 초록색으로 나타낸 것 역시 sleep 을 강조하는 의미이다(Fig. 1A).

대한수면연구학회는 2002년 4월 12일에 대한수면연구회 로 발족하였으며, 2008년 9월 9일에 대한수면연구학회로 학 회 명칭을 변경하여 오늘에 이르고 있다. ${ }^{10}$ 학회의 영문명은 Korean Sleep Research Society이며, 약자는 KSRS이다. 대 한수면연구학회 또한 의사, 치과의사, 수면 관련 분야의 박사 학위 소지자 또는 이에 상응하는 자를 정회원으로 한다. 대한 수면연구학회에서 현재 사용하는 문장은 2013년 제10회 대 한수면연구학회 학술대회에서부터 개정하여 사용하고 있다 (Fig. 1B). 학회의 창립 회원이자 해당 학회의 회장을 역임한 바 있는 성균관대학교 의과대학 홍승봉 교수에 따르면, 학회 의 문장은 큰 원으로 태극 문양으로서 음과 양을 상징하며, 가운데의 흰색의 형체는 잠을 자고 있는 사람의 형상을, 그 리고 그 옆에 뇌파를 형상화한 부분으로 구성되었다(Hong $\mathrm{SB}$, personal opinion). 그리고 한글과 영어로 표시한 학회 의 정식 명칭이 둥근 모양의 문장을 둘러싸도록 고안되었다. 대한수면의학회는 1993년 7월 2일에 대한수면-정신생리 학회(Korean Academy of Sleep and Psychophysiology)로 서 설립되어 2002년 9월부터 대한수면의학회로 학회명을 개 정하였다. ${ }^{11}$ 대한수면의학회의 영문명은 Korean Academy of Sleep Medicine이며, 영문 약자는 KASMED이다. 이 학 회의 정회원은 수면의학 및 연관 분야의 전문의로 규정하고 있다. ${ }^{12}$ 대한수면의학회에서 사용하는 문장은 Fig. $1 \mathrm{C}$ 와 같 으며, 학회 설립 때부터 이 문장을 사용하고 있다. 학회의 문

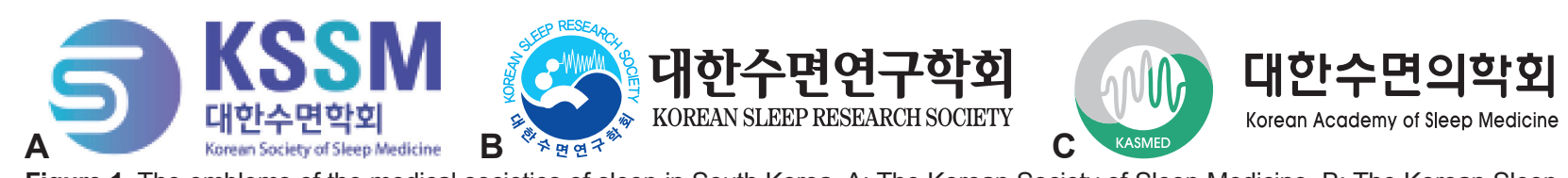

Figure 1. The emblems of the medical societies of sleep in South Korea. A: The Korean Society of Sleep Medicine. B: The Korean Sleep Research Society. C: The Korean Academy of Sleep Medicine. 
장을 살펴보면 바깥쪽 원에 태극 문양이 있고, 가운데 원 안 에는 수면방추체를 형상화한 그림이 위치하고 있으며, 그 아 래에 학회의 이름을 약자(KASMED)로 기입하였다.

\section{우리나라의 수면 관련 학회가 소속된 국제 학회}

세계수면학회는 2016년부터 세계수면협회와 세계수면연 맹이 통합되어 운영되고 있으며, ${ }^{13}$ 이에 대한 세부사항은 해당 학회의 홈페이지에서 자세히 확인할 수 있다. 2020년 현재 약 14,000 명의 회원과 44 개의 연관 학회를 보유하고 있으며, 문 장은 Fig. 2A와 같다. 세계를 아우르는 학회임을 나타내기 위 하여 지구를 형상화하여 오른쪽에 배치하였으며, 태극 문양 은 찾아볼 수 없다. 영문으로 “WORLD SLEEP SOCIETY”를 기입하고 $\mathrm{W}$ 를 특수한 모양으로 디자인하였는데, 학회 사무 국의 답변에 따르면 2001년에 창립된 세계수면협회(WASM) 의 문장을 계승한 것이다(Pack AI, personal opinion). 학회 이름 아래에 있는 "Advancing Sleep Health Worldwide"는 세계수면학회의 사명(mission)이며, ${ }^{14}$ 문장의 아래에는 세계 수면협회와 세계수면연맹의 통합으로써 세계수면학회가 설 립되었음을 명시하고 있다.

아시아수면연구학회는 1994년 6월 도쿄에서 창립되었으 며, ${ }^{15}$ 학회의 연혁과 문장은 해당 학회의 홈페이지에서 확인 할 수 있다. 우리나라의 대한수면학회와 대한수면의학회를 포함하여 10 개의 단체가 회원으로 등록되어 있고, 중국, 홍 콩, 인도, 인도네시아, 이스라엘, 일본, 말레이시아, 파키스탄 등의 수면 관련 학회들이 소속되어 있다. 이 학회는 세계수면 학회의 창립 회원 학회이며, ${ }^{5} \mathrm{Fig} .2 \mathrm{~B}$ 와 같은 문장을 채택하 고 있다. 전체적으로는 아시아(Asia)의 A 모양에 태극 문양 을 삽입하여 음과 양을 표현하였다.

아시아수면학회는 2013년 6월에 창립되었으며, ${ }^{16}$ 이와 관 련한 회의록과 회칙은 해당 학회의 홈페이지에 상세하게 기 록되어 있다. ${ }^{17}$ 우리나라의 대한수면학회 외에 중국, 홍콩, 인도, 인도네시아, 이스라엘, 일본, 말레이시아, 태국, 필리핀, 사우디아라비아, 이란, 베트남, 싱가포르, 오만 등의 관련 학 회들이 소속되어 있다. 아시아수면학회는 2017년부터 세계 수면학회의 회원이다. ${ }^{18}$ 이 학회의 문장은 Fig. $2 \mathrm{C}$ 와 같으며, 이 문장을 고안한 Chang Gung Memorial Hospital의 NingHung Chen에 따르면, 역시 음과 양을 나타내는 태극 문양 에서 고안하였다(Chen NH, personal opinion). 커다란 태극 문양에 영문으로 학회 명칭이 기입된 이 문장은 2015년에 대
한민국 서울의 코엑스에서 열린 업무 회의에서 처음 소개되 었다. ${ }^{19}$

\section{결 론}

본 연구에서 조사한 6 개의 수면 관련 학회들을 설립 연도 순으로 정리하면 대한수면의학회(1993), 아시아수면연구학 회(1994), 대한수면연구학회(2002), 대한수면학회(2006), 아 시아수면학회(2013), 세계수면학회(2016)의 순이다. 이 중에 서 대한수면연구학회와 대한수면학회에서는 창립 후 10여 년 후에 새로운 문장을 채택하여 사용하였다. 이에 따라 각 각의 문장이 채택된 순으로 나열하면 대한수면의학회(1993), 아시아수면연구학회(1994), 대한수면연구학회와 아시아수면 학회(2013), 세계수면학회(2016), 그리고 대한수면학회(2019) 의 순이다.

문장을 그 구성과 내용에 따라서 분류한다면 태극 문양, 뇌파, 그리고 학회의 명칭(문자)으로 나눌 수 있다. 수면 관련 학회 문장의 구성 중 가장 흔히 볼 수 있는 문양은 태극이다. 우리나라의 수면 관련 학회 3개 중에서 대한수면연구학회와 대한수면의학회에서는 태극 문양을 채택하고 있으며, 이는 아시아수면학회와 아시아수면연구학회에서도 확인할 수 있 다. 수면 관련 학회의 문장을 구성하는 과정에서 인간의 수 면을 연상하는 테마로서 낮과 밤을 떠올린다면, 그다음은 음 과 양의 상징인 태극으로 이어지는 자연스러운 연상의 흐름 으로 사료된다. 그런데 이 학회들의 문장을 자세히 들여다보 면, 태극 문양을 음과 양이 나뉘는 방향에 따라 크게 두 가지 로 나눌 수 있다. 아래와 위로 배치된 경우로서 대한수면연 구학회와 대한수면의학회가 있으며, 왼쪽과 오른쪽으로 나 뉘는 경우로서 아시아수면학회와 아시아수면연구학회로 분 류할 수 있다. 통상적으로 우리나라에서는 태극기에서 사용 하는 태극을 모티브로 하여 음과 양이 각각 아래와 위로 나 누어지는 구성을 채택한 것으로 보이며, 아시아수면학회와 아시아수면연구학회에서 태극과 두 개의 점으로 구성된 문 양을 채택한 것은 중국 문화의 영향으로 사료된다. 한편, 문 장에서 뇌파를 형상화한 경우는 대한수면연구학회와 대한 수면의학회의 두 개 학회에서 관찰할 수 있는데, 대한수면연 구학회에서는 뇌파를 단순화하여 삽입하였고 대한수면의학 회에서는 수면방추체를 형상화하였다. 문장에 뇌파를 삽입 한 것은 뇌파 검사를 수면의 중요한 지표로 인식하고 있음을

\section{A WORLD SLEEP SOCIETY \\ B A. Asian Sleep Research Society


시사한다. 세부적으로는 대한수면연구학회에서 채택한 뇌 파는 특정 수면 단계와 관련이 없는 '뇌파'이며, 대한수면의 학회의 문장에는 N2 수면에서 관찰할 수 있는 수면방추체가 있다. 우리나라의 3 개 학회에서는 공통적으로 학회의 한글 명칭과 영문 명칭을 병기하고 있다. 또한 모든 학회는 회칙 에서부터 학회의 약자를 정확히 규정한다. 모든 학회가 영문 이름의 두문자어(acronym)로 학회 명칭의 약어를 정하는 가 운데, 오직 대한수면의학회에서만 KASMED로 학회의 약어 를 따로 정하였다. 특히 대한수면연구학회에서는 학회의 원 형 문장에 한글로 된 학회의 정식 명칭을 기입하여, 본 연구 에서 수집한 6 개의 수면 관련 학회 가운데 유일하게 한글이 문장에 포함된 형태를 가지고 있다(Fig. 1B).

본 연구에서 수집한 모든 수면 관련 학회에서는 문장에 학회 명칭의 문자를 분리하지 않고 다양한 형태로 혼합된 디 자인을 채택하였다. 대한수면학회는 학회 이름의 영문 약어 $(\mathrm{KSSM})$ 와 한글 이름, 그리고 영문으로 학회 이름을 기입한 문자는 문장과 따로 분리되어 있는 형태이지만, 문장에 sleep 의 $\mathrm{S}$ 를 포함한 점은 문자가 혼합된 문장의 형태로 볼 수 있 다. 대한수면연구학회의 문장은 가운데의 문장 주변으로 영 문과 한글로 학회의 정식 이름을 기입하여 문자가 혼합된 문 장의 형태이다. 대한수면의학회에서도 문장에 표현된 수면방 추체의 아래에 학회 이름을 약자(KASMED)로 기입하여 문 자가 혼합된 문장의 형태를 하고 있다. 세계수면학회의 문장 은 지구를 형상화한 것으로 볼 수도 있지만, 실제로 학회 활 동과 관련된 모든 문서나 웹사이트에서 "WORLD SLEEP SOCIETY"를 표기하고 있으며, 지구를 형상화한 배경에 특 수한 디자인의 W를 사용하기도 한다. 따라서 세계수면학회 의 문장은 문자가 혼합된 형태로 보아야 한다. 아시아수면연 구학회의 문장은 하나의 그림으로 보이기 때문에 학회 명칭 의 문자 없이 문장만을 단독으로 표현한 것이라 볼 수도 있 겠지만, Fig. $2 \mathrm{~B}$ 를 자세히 보면 전체적으로는 알파벳 $\mathrm{A}$ 에서 하나의 획을 태극 문양으로 대체한 것이므로 문자가 혼합된 문장의 형태라고 볼 수 있겠다. 아시아수면학회의 문장은 태 극 문양과 학회 명칭이 나란히 배치되어 문장과 문자가 독립 된 형태라고 생각할 수도 있겠지만, 태극 문양 단독으로 학 회를 떠올리기는 어렵고 태극 문양이 학회를 상징하는 독창 적인 디자인이라고 보기 힘들다. 게다가 학회 이름의 약자 중 에서 $\mathrm{A}$ 와 태극 문양을 겹치게 만들어서 실제로는 모든 문서 나 웹사이트에서 Fig. $2 \mathrm{C}$ 전체를 사용하고 있으므로, 문장과 문자가 혼합된 형태라고 보는 것이 타당하다.

단체를 나타내는 여러 가지 방법 중에서도 문장은 상징적 인 표지이며, 단순한 대표성을 넘어 다양한 의미가 포함되어 있다. 즉, 해당 단체가 대표하고자 하는 집단의 정체성을 나
타내고자 노력하고 있으며, 지향하는 방향을 문장에 형상화 하여 담아내고 있다. 이에 따라 우리나라 정부 등의 공공기 관에서는 상징이나 문장의 세부적인 색깔, 문장의 크기, 글 자의 모양과 위치 등과 같이 세부적인 사항을 적시하는 등의 노력을 기울이고 있다. 하지만 저자가 수면 관련 학회의 문 장을 조사한 범위 안의 국내 · 외 수면 관련 학회에서, 문장의 세부적인 디자인을 적시하는 곳은 찾아볼 수 없었다. 학회의 문장과 같은 상징에 대한 기록이 부실하여, 이메일 혹은 전 화로 해당 학회의 문장에 대해서 문의했을 때 그 의미를 정 확하고 자세하게 답변하는 국내 학회가 없었다. 비단 우리나 라의 수면 관련 학회뿐만 아니라, 본 연구에서 조사한 어떤 학회에서도 웹사이트나 회칙에서 학회 문장의 유래, 구체적 인 형식, 그리고 그 의미를 명쾌하게 답변한 곳은 없었으며, 학회 문장의 저작권을 명시한 곳도 찾아볼 수 없었다. 여러 연구자들의 노력에 힘입어 우리나라뿐만 아니라 세계적으로 도 수면의학은 눈부시게 발전하고 있다. 수면의학의 학문적 발전만큼이나 문장과 로고 등 학회의 상징은 꼭 필요한 부분 이지만 지금까지 비교적 관심이 부족했다. 이것은 20년에서 30년에 이르는 우리나라 수면 관련 학회의 발전에서도 중요 한 부분임에는 이견이 없을 것이다. 학회에서는 역사와 정체 성에 대한 관리와 노력이 필요하며, 여기에는 학회 문장과 로 고 등의 상징에 대한 관리가 필요하다.

본 연구에 포함된 대한수면학회, 대한수면연구학회, 대한 수면의학회는 적어도 연 1회 이상의 한국학술지인용색인 (KCI) 등재학술지를 발간하고 연구자들에게 널리 알려진 단 체이며 일정 형식의 회칙을 갖고 있기 때문에 공인된 학회로 받아들일 수 있어서 분석의 대상으로 선정하였다. 또 세계적 으로 활동하는 수많은 수면 관련 학회나 단체를 모두 조사 하기에는 무리가 있어, 우리나라의 3개 학회가 참여하고 있 는 국제 학회를 역으로 추적하여 선정하게 되었다. 따라서 본 연구에서 다루는 문장이 수면에 대한 우리나라 국민들이나 세계인의 수면에 대한 인식을 대표한다고 말하기는 어렵다. 그럼에도 불구하고, 본 연구는 수면 관련 학회의 문장을 주 제로 한 국내 최초의 연구로서 학회가 지니는 역사와 정체 성에 대해서 의학적 부분과 의학 외적 부분을 연결시켜 고 찰을 시행한 점에서 의미가 있다고 할 수 있겠다. 아무쪼록 이 연구가 우리나라의 수면의학이 더 관심받고 그 저변이 확 대되는 계기가 되어 수면의학의 발전에 도움이 되길 바란다.

\section{Conflicts of Interest}

The authors have no potential conflicts of interest to disclose.

\section{ORCID iDs}

Keun Tae Kim https://orcid.org/0000-0002-7124-0736 Won Chul Shin https://orcid.org/0000-0003-3044-9397 
Jee Hyun Kim

https://orcid.org/0000-0002-8142-4635

Yong Won Cho

https://orcid.org/0000-0002-6127-1045

\section{Author Contributions}

Conceptualization: Keun Tae Kim. Data curation: Keun Tae Kim, Yong Won Cho. Formal analysis: all authors. Investigation: Keun Tae Kim, Won Chul Shin, Jee Hyun Kim. Methodology: Yong Won Cho. Project administration: Keun Tae Kim, Yong Won Cho. Supervision: Yong Won Cho. Visualization: Keun Tae Kim. Writing_original draft: Keun Tae Kim. Writing-review \& editing: all authors.

\section{Funding Statement}

None.

\section{Acknowledgments}

The authors wish to thank and acknowledge the help of Seung Bong Hong (currently serving as the president of the Korean Neurological Association) in investigating the meaning of the emblems and the history of the sleep societies.

\section{REFERENCES}

1. National Institute of Korean Language. Pyojun-gugeo-daesajeon: Hakhoi [Internet]. Seoul: National Institute of Korean Language [cited 2021 Jun 7]. Available from: https://stdict.korean.go.kr/search/searchResult. do? pageSize $=108$ searchKeyword $=\% \mathrm{ED} \% 95 \% 99 \% \mathrm{ED} \% 9 \mathrm{~A} \% 8 \mathrm{C}$.

2. Stancliffe. Of sleep; its utility, causes, its varieties, perturbances, defect, excess; of their irregularities, and of the rationale, and cause of dreams; translated from Dr. Gregory's conspectus medicinœ. Med Phys J 1810; 24:279-285.

3. Park BS, Kim IH, Park KM. Top 100 cited articles on sleep medicine: a bibliometric analysis. Eur Neurol 2020;83:111-120. https://doi.org/ 10.1159/000507393.

4. World Sleep Society. About us [Internet]. Rochester, MN: World Sleep Society [cited 2021 Mar 15]. Available from: https://worldsleepsociety. org/about/.

5. World Sleep Society. Current associate society members [Internet]. Rochester, MN: World Sleep Society [cited 2021 Mar 15]. Available from: https://worldsleepsociety.org/membership/societymembership/ membersocieties/.

6. National Institute of Korean Language. Pyojun-gugeo-daesajeon: Munjang [Internet]. Seoul: National Institute of Korean Language [cited 2021 Jun 7]. Available from: https://stdict.korean.go.kr/search/ searchView.do?word_no=126673\&searchKeywordTo=3.
7. The Government of the Republic of Korea. Korean government symbol system design guide 2017 revision [Internet]. Sejong: Minstry of Enviroment; 2017 [cited 2021 Mar 21]. Available from: https://me. go.kr/home/file/readDownloadFile.do?fileId=142132\&fileSeq=2.

8. Ministry of Culture, Sports and Tourism. Symbol [Internet]. Sejong: Ministry of Culture, Sports and Tourism; 2016 [cited 2021 Mar 3]. Available from: https://www.mcst.go.kr/english/ministry/symbol/symbol.jsp.

9. Korean Society of Sleep Medicine. Rules [Internet]. Seoul: Korean Society of Sleep Medicine [cited 2021 Jan 15]. Available from: http:// www.sleepmed.or.kr/content/about/rule.html.

10. Korean Sleep Research Society. Rules [Internet]. Daegu: Korean Sleep Research Society [cited 2021 Mar 25] Available from: https://www. sleepnet.or.kr/introduce/rule.

11. Korean Academy of Sleep Medicine. History [Internet]. Seoul: Korean Academy of Sleep Medicine [cited 2021 Feb 1] Available from: https:// www.sleep.or.kr/html/?pmode=history.

12. Korean Academy of Sleep Medicine. Rules [Internet]. Seoul: Korean Academy of Sleep Medicine; 2020 [cited $2021 \mathrm{Feb}$ 15]; Available from: https://www.sleep.or.kr/html/?pmode=rule.

13. World Sleep Society. Frequently asked questions - bylaws [Internet]. Rochester, MN: World Sleep Society; 2016 [cited 2021 Mar 15]. Available from: https://worldsleepsociety.org/about/bylaws/faq/.

14. World Sleep Society. 2020 Annual report [Internet]. Rochester, MN: World Sleep Society; 2020 [cited 2021 Mar 15]. Available from: https:// worldsleepsociety.org/about/annualreport/.

15. Cherasse Y. Overview of sleep and sleep medicine in Asian countries. Sleep Biol Rhythms 2011;9(Suppl 1):84-89. https://doi.org/10.1111/ j.1479-8425.2010.00468.x.

16. Asian Society of Sleep Medicine (ASSM). Minutes of first meeting to initiate the foundation [Internet]. Taipei: Asian Society of Sleep Medicine; 2013 Jun 8 [cited 2021 Mar 31]. Available from: http://www. asiansleep.org/about\%20assm/meeting_text.php?meeting_id=2.

17. Asian Society of Sleep Medicine (ASSM). Minutes of annual meetings \& bylaw [Internet]. Taipei: Asian Society of Sleep Medicine; 2014 Mar 13 [cited $2021 \mathrm{Mar}$ 15]. Available from: http://www.asiansleep.org/index.php.

18. World Sleep Society. Asian Society of Sleep Medicine [Internet]. Rochester, MN: World Sleep Society; 2020 [cited 2021 Mar 15] Available from: https://worldsleepsociety.org/membership/societymembership/ membersocieties/asian-society-of-sleep-medicine/.

19. Asian Society of Sleep Medicine (ASSM). ASSM business meeting in Seoul 2015 [Internet]. Taipei: Asian Society of Sleep Medicine; 2015 [cited 2021 Mar 15]. Available from: http://www.asiansleep.org/about\%20 assm/meeting_text.php?meeting_id=3. 